\title{
Structural synthesis of Euclidean platform robot manipulators with variable general constraints
}

\author{
Rasim Alizade*, Fatih Cemal Can, Erkin Gezgin \\ Mechanical Engineering Department, Izmir Institute of Technology, Urla 35437, Izmir, Turkey
}

Received 16 November 2006; received in revised form 11 May 2007; accepted 23 November 2007

Available online 14 January 2008

\begin{abstract}
In this paper, new method for structural synthesis of Euclidean platform robot manipulators with variable general constraints (EPRM) is presented. Three dimensional motion of the base moving platform is generated by the motion of dyads on the Euclidean planes. Each dyad is connected to the moving base platform by universal, spherical or spherical in torus $\left(\mathrm{S}_{\mathrm{t}}\right)$ kinematic pairs. This allows solving the structural synthesis of Euclidean robot manipulators with various DoF and different platform motions. New structural formulations with variable general constraints for the platform motion and the mobility of robot manipulators are presented. The new structural classification of simple structural groups with variable general constraints including platforms, hinges, legs and branch loops from different subspaces and space is also introduced. Moreover, new method for structural synthesis of serial, parallel and serial-parallel EPRM is illustrated along with examples.
\end{abstract}

(c) 2007 Elsevier Ltd. All rights reserved.

\section{Резюме}

В работе представлен новый метод структурного синтеза Евклидовых робот манипуляторов параллельной структуры. На основе движения основной платформы и движения диад в Евк.лидовых плоскостьях разработан новый метод структурного синтеза. Каждая диада соединяется посредством (сферической пары- 3 подвижная пара, либо сфера в торосе- 4 подвижная пара, либо сфера в цилиндре- 4 подвижная пара) кинематических пар с основной движущейся платформой. Этот метод позволяетрешать задачи структурного синтеза Евклидовых робот манипуляторов с различными степенями свободы и движения платформы. Представлены новые логические структурные формулы движения платформы и степени свободы робот манипуляторов. Новый метод структурного синтеза Евклидового платформенного типа робот манипуляторов параллельной структуры иллюстрируются примерами.

(c) 2007 Elsevier Ltd. All rights reserved.

Keywords: Structural synthesis; Variable general constraints; Branch loops; Motion of platform; Euclidian manipulators

\footnotetext{
* Corresponding author. Tel.: +90 232750 6544; fax: +90 2327507890.

E-mail addresses: alizada_rasim@hotmail.com, rasimalizade@iyte.edu.tr (R. Alizade).
} 


\section{Introduction}

Study of structural synthesis always be in a state of development during the last centuries. Due to this development, many investigations on this subject are discussed in literature. Detailed and recent review about kinematic structure of mechanisms was introduced by Mruthyunjaya [1] in 2003. In his review, pattern of growth of literature on kinematic structure over four decades is shown. In 2005, Gogu [2] introduced a mobility analysis of translational parallel robot manipulators, which is different from previous mobility analyses. After this study, in 2006, Alizade et al. [3] reviewed the history of degrees of freedom analysis and structural synthesis formulations in a table that also includes the names of authors, publication dates and commentaries. Furthermore, a new structural synthesis formulation of Cartesian robot manipulators is presented in the same investigation.

Denavit and Hartenberg [4], Sheth et al. [5], and Khalil et al. [6] introduced the link and joint parameters, which allow the mathematical modeling of robotic mechanical systems. Methodological structural synthesis of serial parallel manipulators is introduced by Alizade and Bayram [7]. Creation of CAD structural system by using topological description method of kinematic chains and classification of robotic systems was done by Roth [8]. Graph theory of structural synthesis and analysis of mechanisms have been investigated by using the method of intuition and inspection method, Crossley [9] and Woo [10], by using the concept of transformation of binary chains for the structural synthesis of kinematic chains with up to 10 links and 3 DoF, Mruthyunjaya [11-13], by using the development of structural Assur groups, Manolescu [14-16], by using robotic system application, Merlet [17] and by using CAD strucutural synthesis of planar kinematic chains, Hwang et al. [18]. Topological structure, description and classification of industrial robots of different levels have been presented by Mitrouchev [19] by using the number of closed loops. Method on the concept of loop formation, which obviates the necessity of the test for isomorphism, is presented by Rao et al. [20]. Zhoo et al. [21] proposed new concept of configuration degrees of freedom (CDoF) that can form a theoretical base for analyzing the mobility, singularity and stability of mechanisms.

Huang and $\mathrm{Li}$ [22] proposed a general methodology for type synthesis of lower mobility parallel manipulators by using screw theory. By the help of proposed method, they presented three novel lower mobility parallel manipulators with 3, 4 and 5 DoF. In the light of well known Tricept robot, Huang et al. [23] designed a new hybrid robot manipulator named Trivariant. They also compared their design with Tricept robot according to cost and kinematic performance. The new parallel manipulator family, where at least one leg contains a planar four-bar parallelogram has been presented by Liu et al. [24]. Some fully parallel mechanisms with two to six DoFs, where at least one leg consists of a planar four-bar parallelogram are intended for pure translation in planar, high or improved rotational capability and better stiffness [25]. Fang and Tsai [26] developed a systematic approach of structural synthesis by using screw theory. They enumerated limb structures for constructing 4-DoF or 5-DoF parallel manipulators according to reciprocity of limb twist system and wrench system. The Lie group of rigid body displacements is represented by operator including screw or twist. The screw system has a Lie algebraic structure that represents all possible displacements. In the study of Herve [27], it is shown that mathematical representation of the connection between any pair of bodies obtained through two operations, the composition and the intersection of mechanical bonds. New manipulator with 3 DoF motions of a platform where three limbs generates subsets of possible displacements, Lie subgroup of Schoenflies motions, is illustrated.

In current study, new parallel manipulators, which are classified as parallel Euclidean platform robot manipulators are introduced. After structural synthesis preliminaries, Euclidean motions are explained to describe new EPRM. New structural formulas of parallel and serial platform Euclidean robot manipulators with variable general constraints are introduced. Furthermore, parallel multiplatform Euclidean robot manipulators and their structural classification with variable general constraints of branch loops are presented. Also, structural parameters, kinematic structures, motion of platforms and 3D drawings of new manipulators are depicted in tables.

\section{Structural synthesis preliminaries}

Structural synthesis of mechanisms is one of the main branches of the fundamental Mechanisms and Machine Science. Structural synthesis is a methodology that is used to generate all structures with desired kinematic performance. 
The investigations on structural synthesis of mechanisms are generally studied in sub-categories as geometrical and kinematic structural synthesis. The purpose of geometrical structural synthesis is to create data foundation to discover particular geometrical features and optimum structures by;

- Further development theory of degrees of freedom of mechanisms with variable general constraints and motion of platforms.

- Generating kinematic chains for hinges, branch-loops, and legs of platforms to create simple structural groups.

- Linking simple structural groups to the actuators of manipulators.

- Linking simple structural groups with variable general constraint parameters to the moving platform and ground or actuators of parallel Cartesian robot-manipulators.

- Creating new EPRM with variable general constraints in space or subspaces.

- Creating modular systems with multimobility using successive layers of serial trees and parallel manipulators.

- Computer-aided structural synthesis.

On the other hand, Kinematic structural synthesis focuses on the following problems;

- Generation of the branches and legs of parallel manipulators by describing the axis of kinematic pairs and links, also joint and link construction parameters.

- Identifying angular and linear conditions for over constraint mechanisms.

- Rearranging the leg configurations of parallel manipulators in such a way that it will be easier to carry out the forward and the inverse tasks.

\section{Structural synthesis of Euclidean platform robot manipulators with variable general constraints}

Serial robots are limited in the number of possible mechanical structures; however, there is a variety of possible parallel robots that are constructed from the branch loops with variable general constraints, multiple platforms, hinges and legs. Note that, the overall performance of these robots can be affected by the topology of their structures.

\subsection{Motion in Euclidean planes}

In this study, Euclidean planes are utilized to obtain Euclidean motions. Euclidean motion of $\mathbb{R}^{2}$ is an affine transformation whose linear part is orthogonal [28]. Examples of affine transformation can be given as geometric contractions, expansions, dilations, reflections, rotations, shears, similarity transformations, spiral similarities, translations and their combinations. However, rotations and translations are enough for our study due to rigid links and platforms of the manipulators. The new proposed Euclidean manipulators have several legs, which create Euclidean motions on their own Euclidean planes.

In order to obtain Euclidean plane motion in the design, legs of manipulators are selected as dyads. These dyads can be RR, PR, RP and PP chains as shown in Fig. 1. Note that, point G of each leg is connected to the platform by universal, spherical or spherical-torus pairs. Position of point $G$ with respect to the fixed reference frame (Fig. 1) defines the curve of one point of the platform in the reference Euclidean plane. The motion of the platform can be defined by minimum three independent curves of three platform points moving on three Euclidean reference planes.

Note that, a kinematic pair with $4 \mathrm{DoF}$ is also introduced as spherical torus pair $\left(\mathrm{S}_{\mathrm{t}}\right)$, which consists of three rotations and one circular translation, (Fig. 2). The name of the kinematic pair comes from its torus workspace that is drawn by using Mathematica computer software, after the kinematic analysis of the pair by Denavit-Hartenberg convention.

\subsection{Structural formula of parallel and serial Euclidean platform robot manipulators}

It is clear that freely moving platform in three dimensional space has six degrees of freedom. This DoF is related to the location of the platform in space with respect to the reference frame. Location of the platform 


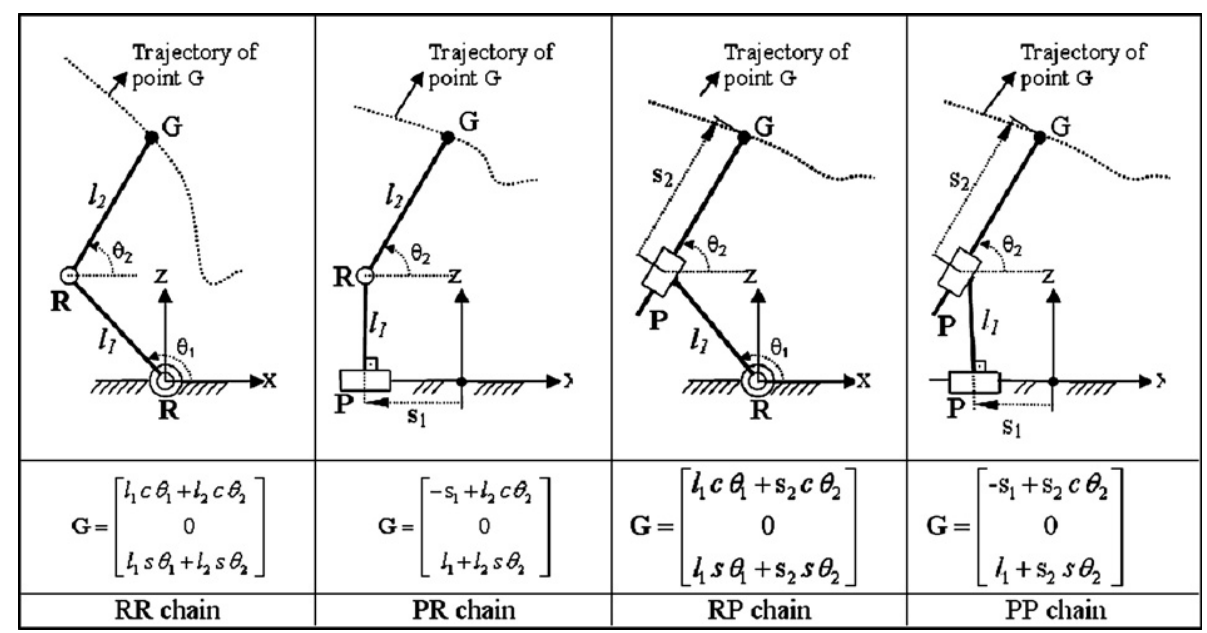

Fig. 1. Four possible dyads for the legs of EPRM.
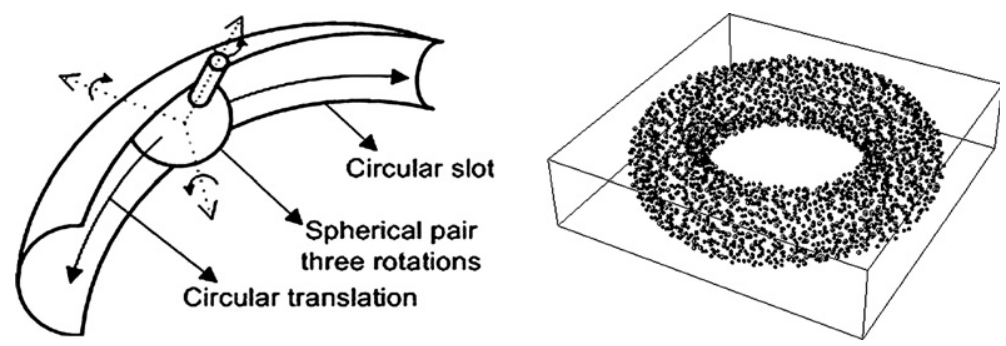

Fig. 2. Spherical-torus $\left(\mathrm{S}_{\mathrm{t}}\right)$ kinematic pair and its workspace.

defines both position and orientation of the moving coordinate system, which is attached to the moving platform, with respect to the fixed frame as shown in (Fig. 3a), where $u, v$ and $w$ are the axes of the moving frame and define the direction cosines of the moving frame that consists of three rotations and $\rho$ has three translational component $\left(\rho_{x}, \rho_{y}, \rho_{z}\right)$, which indicates the origin of the moving frame with respect to the fixed frame. If two moving platforms are connected by hinge (revolute pair), DoF of the serial moving platforms is increased to seven (Fig. 3b). If the number of hinges between the platforms is more than one (Fig. 3c), DoF of the serial moving platforms can be calculated as,

$$
M_{\mathrm{SP}}=\lambda+j_{h}
$$

where $\lambda$ is the number of independent parameters describing the positions and orientations of any rigid body in space or subspaces and $j_{h}$ is the number of hinges between platforms.

If the entire legs of the serial platform manipulators are connected to the moving serial platforms and to the ground, mobility of the kinematic chains of the legs can be defined as,

$$
M_{l}=\sum_{l=1}^{c_{l}}\left(f_{l}-\lambda_{l}\right)
$$

where $\sum_{l=1}^{c_{l}} f_{l}$ is the total DoF of all the kinematic pairs on the legs $c_{1}$, and $\lambda_{1}$ is the general constraint of each $\operatorname{leg}\left(\lambda_{1}=2, \ldots, 6\right)$.

The combination of Eqs. (1) and (2) results in the general structural formula of serial platform robot manipulators as 
a

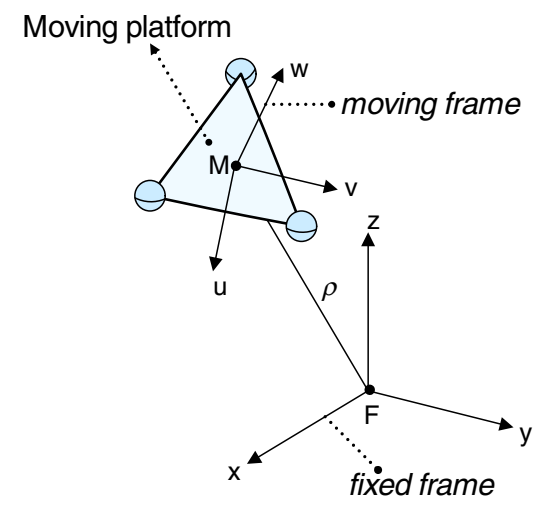

b

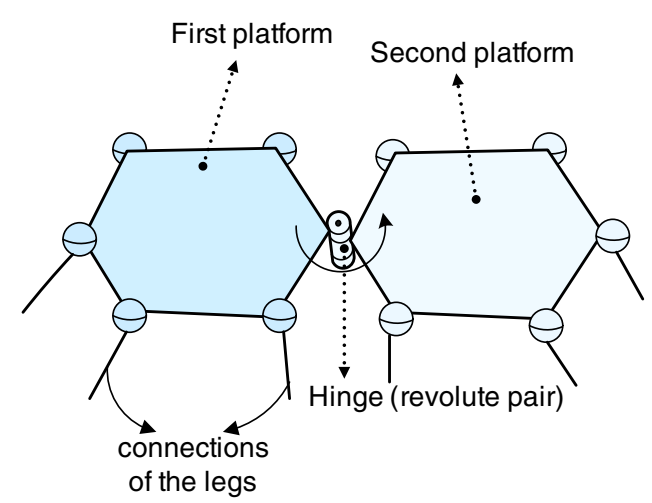

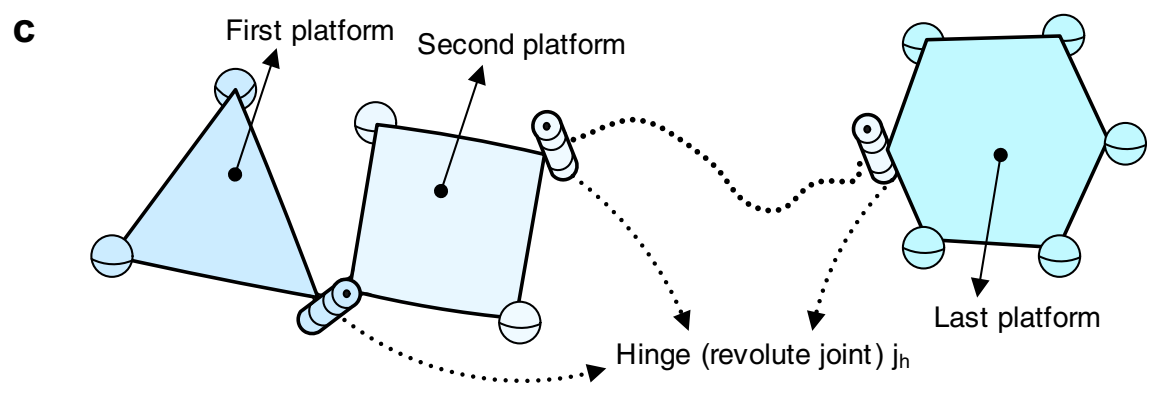

Fig. 3. (a) Six independent quantities of the moving platform. (b) Two platform connected by hinge (revolute pair). (c) Several numbers of serial moving platforms.

$$
M=\lambda+j_{h}+\sum_{l=1}^{c_{l}}\left(f_{l}-\lambda_{l}\right)
$$

If the number of moving platforms is unity in space $\left(\lambda=6, j_{h}=0\right)$, Eq. (3) will be reduced to the structural formula of parallel manipulators as

$$
M=6+\sum_{l=1}^{c_{l}}\left(f_{l}-\lambda_{l}\right)
$$

The motion of one platform on the parallel robot manipulators can be described by

$$
m_{p}=\lambda+c_{l}+\sum_{l=1}^{c_{l}}\left(d_{l}-D\right)
$$

where $D$ is the number of dimensions of vectors in the reference frame $\left(D\right.$ is three for space $\left(\mathbb{R}^{3}\right)$, and two for plane $\left(\mathbb{R}^{2}\right)$ ), and $d_{1}$ is the number of dimensions of vectors in subspaces of the legs [3]. Also motion of two or more serial platforms can be formulated as,

$$
m=\lambda+c_{l}+\sum_{l=1}^{c_{l}}\left(d_{l}-D\right)+j_{h}
$$

Using the structural formulas, Eqs. (3) and (4), we can calculate the mobility of parallel or serial Euclidean robot manipulators, while Eqs. (5) and (6) can be used to describe the motions of platforms related to them.

Example 1. Design a parallel Euclidean robot manipulator with $c_{1}=5, M=5$, and $\lambda_{1}=6$. Find both the number and kind of kinematic pairs on each leg by solving the problem of structural synthesis.

By using Eq. (4), total DoF of kinematic pairs of the legs can be calculated as, $\sum_{l=1}^{c_{l}} f_{l}=M+$ $\sum_{l=1}^{c_{l}} \lambda_{l}-6=5+30-6=29$. So that, in the designed manipulator, one leg will consist of five kinematic pairs 
Table 1

New parallel Euclidean platform robot manipulators

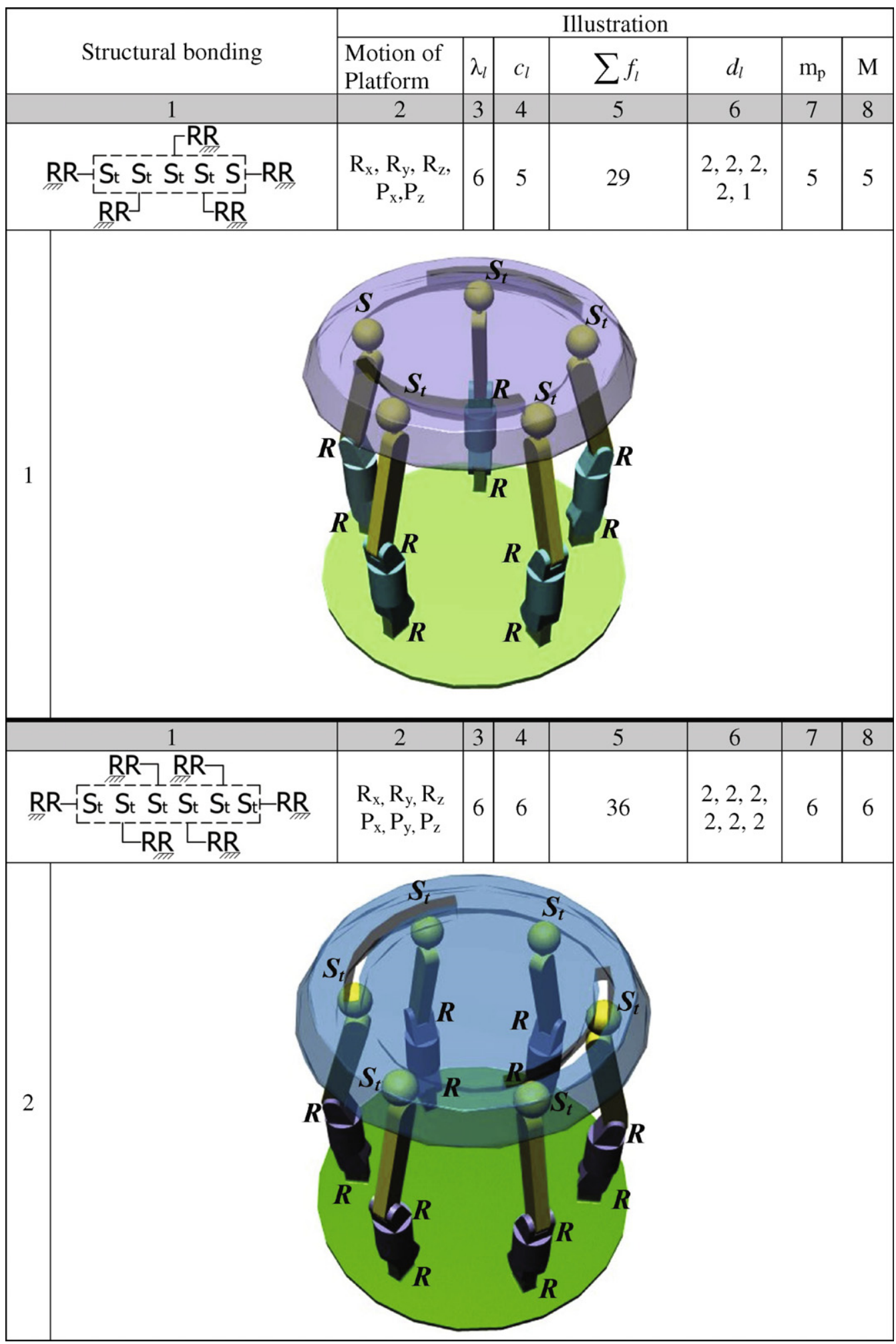


Table 1 (continued)

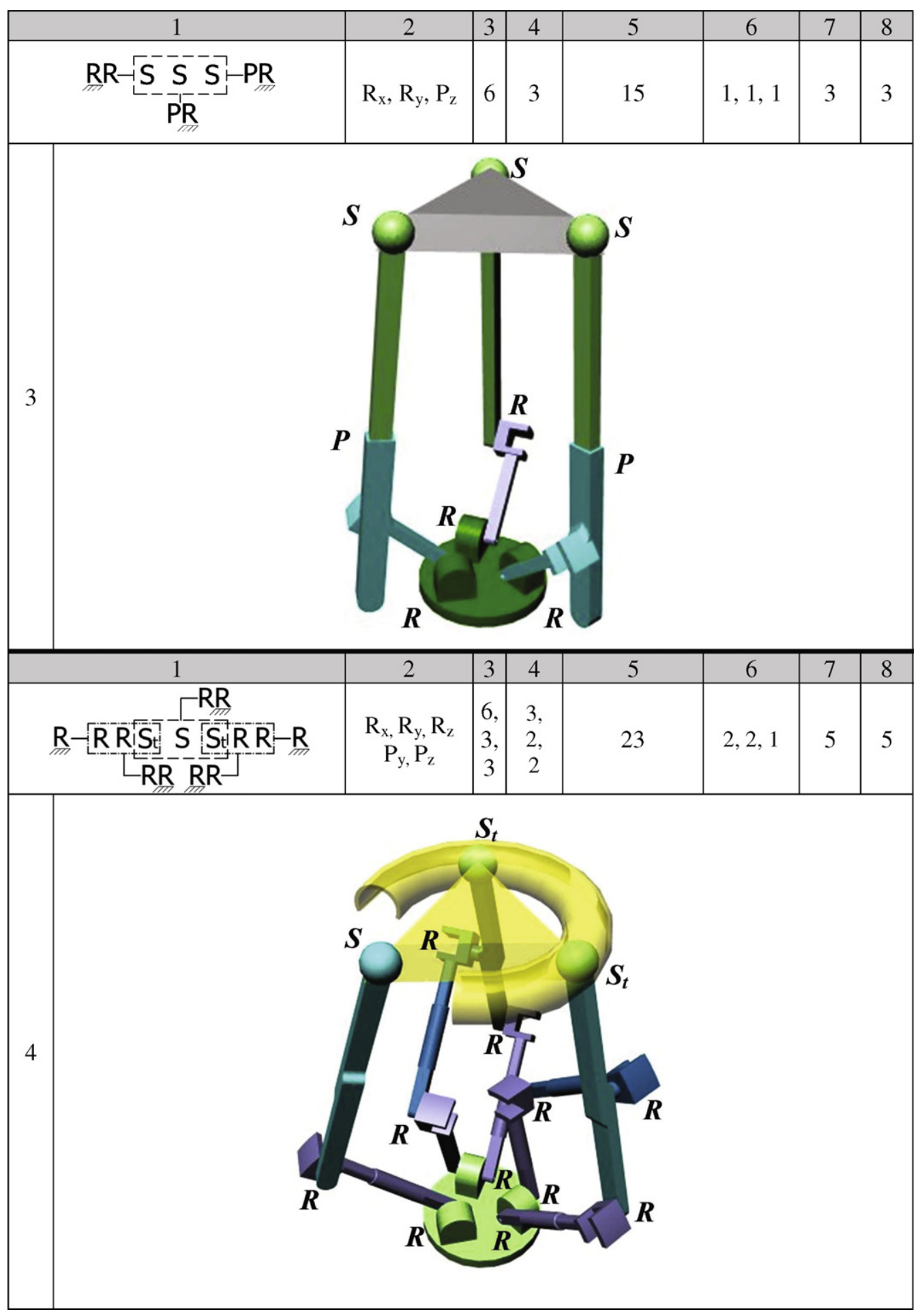


and the remaining legs will consist of six kinematic pairs with one degrees of freedom, $j_{l}=c_{l}^{-1} \sum_{l=1}^{5} f_{l}=5(4)$. Using exchangeability of kinematic pairs and conditions of Euclidean robot manipulators, the design can be improved for the mentioned purpose. Kinematic structure, structural parameters and structural bonding of this robot manipulator can be seen in Table 1.1.

By using the same procedure of structural synthesis, parallel manipulators with different structural parameters can be generated. Some of these new manipulators are shown in Tables 1.1-1.4. Elements of the structural bonds can be illustrated as;

Dashed rectangle (r-a-1

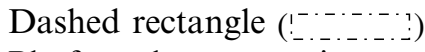
Platform-leg connections
: describes general platforms of moving kinematic chains with hinges and branches

: platforms on the legs

: connection of the pairs on platforms with the remaining pairs of the legs

Fixed frame (77T/ $)$

Example 2. Design a serial Euclidian robot manipulator with $c_{1}=6, M=6$, and $\lambda_{1}=6$. By using these parameters, just two serial platform manipulators with two rectangular platforms or with one triangular and pentagonal platforms can be designed. Now let us take the first one into consideration.

Using Eq. (3), total number of kinematic pairs on the legs can be found as, $\sum_{l=1}^{c_{l}} f_{l}=M+\sum_{l=1}^{c_{l}} \lambda_{l}-$ $\lambda-j_{h}=6+6 \cdot 6-6-1=35$. Each leg will consist of five pairs and the remaining five pairs can be placed to any legs, $j_{l}=c_{l}^{-1} \sum_{l=1}^{6} f_{l}=5(5)$. Kinematic structure of this robot manipulator is shown in Table 2.1. Kinematic structure and the structural bonding of the serial Euclidean platform robot manipulator with $\left(c_{1}=4\right.$, $M=4, j_{h}=1$ and $\lambda_{1}=6$ ) are shown in Table 2.2.

\subsection{Structural formula of Euclidean platform robot manipulators that include branch-loops with variable general constraints}

As mentioned in the previous section, a moving platform has six DoF in space (Fig. 3a). Now consider several parallel platforms connected to each other by branch-loops. Each branch-loop with DoF greater or equal to one can be selected from space or any subspaces. DoF of kinematic chains is equal to the summation between the DoF of base platform, and the DoF of each branch-loop. For instance, if two platforms are connected by a planar loop $(\lambda=3)$ that consists of four revolute joints (Fig. 4a), total DoF of kinematic chains will be seven due to the fact that planar loop gives only one DoF to the system. If several number of platforms are connected by several branch-loops with variable general constraints (Fig. 4b), total DoF of parallel moving platforms can be calculated as,

$$
M_{\mathrm{PP}}=\lambda+\sum_{L=1}^{n}\left(f_{L}-\lambda_{L}\right)
$$

where $n$ is the number of branch-loops, $\lambda_{L}$ is the number of independent, scalar, loop-closure equations associated with each independent loop, $\sum_{L=1}^{n} f_{L}$ is the total DoF of kinematic pairs on the branch-loops.

The general mobility formula of the parallel robot manipulators can be generated by using the combination of Eqs. (2) and (7), as

$$
M=\lambda+\sum_{L=1}^{n}\left(f_{L}-\lambda_{L}\right)+\sum_{l=1}^{c_{l}}\left(f_{l}-\lambda_{l}\right)
$$

Also the motion of parallel platforms with branch-loops can be formulated as,

$$
m=\lambda+c_{l}+\sum_{l=1}^{c_{l}}\left(d_{l}-D\right)+\sum_{l=1}^{c_{l}}\left(f_{L}-\lambda_{L}\right)
$$


Table 2

New serial Euclidean platform robot manipulators that include a hinge

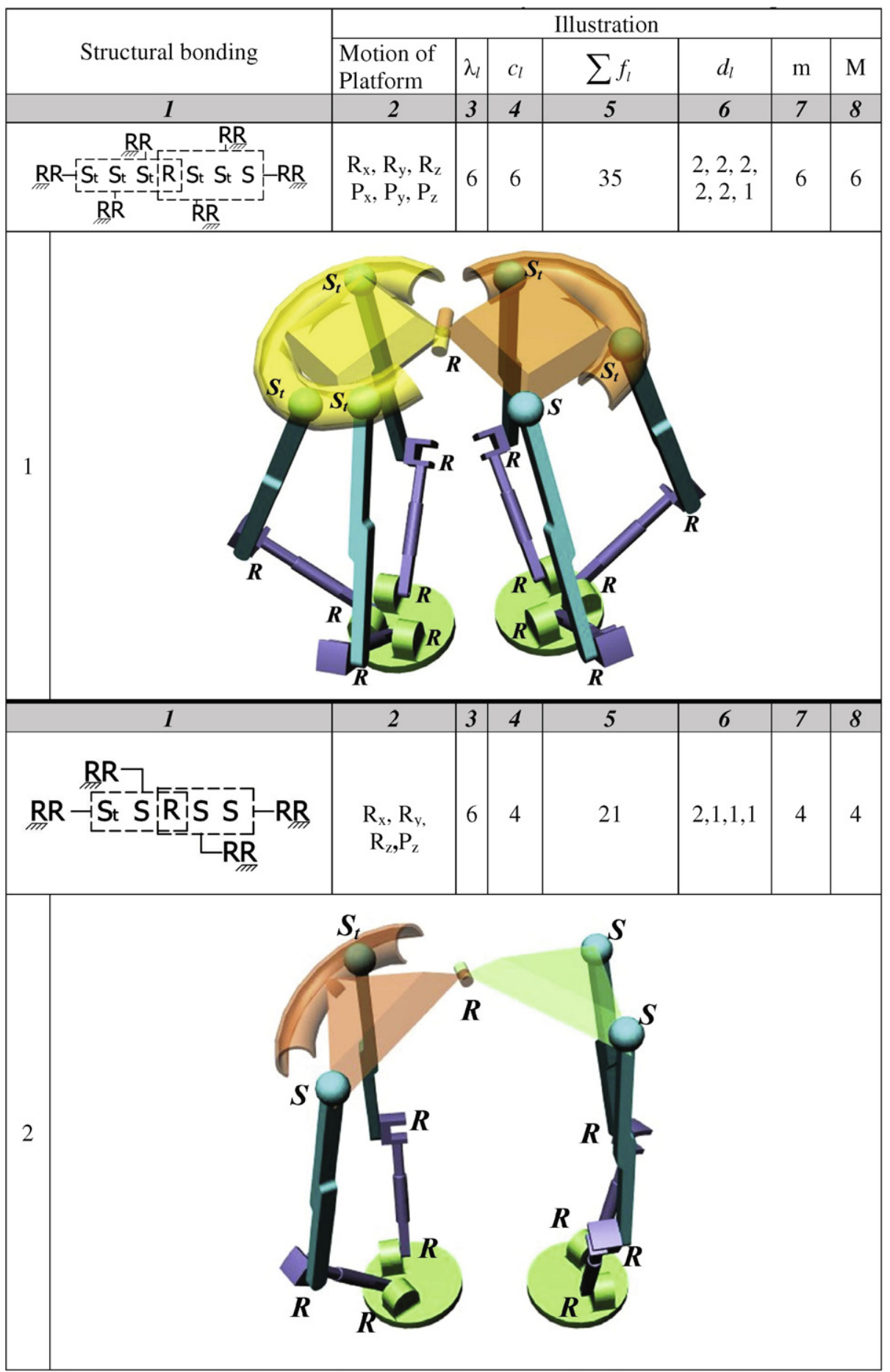


a
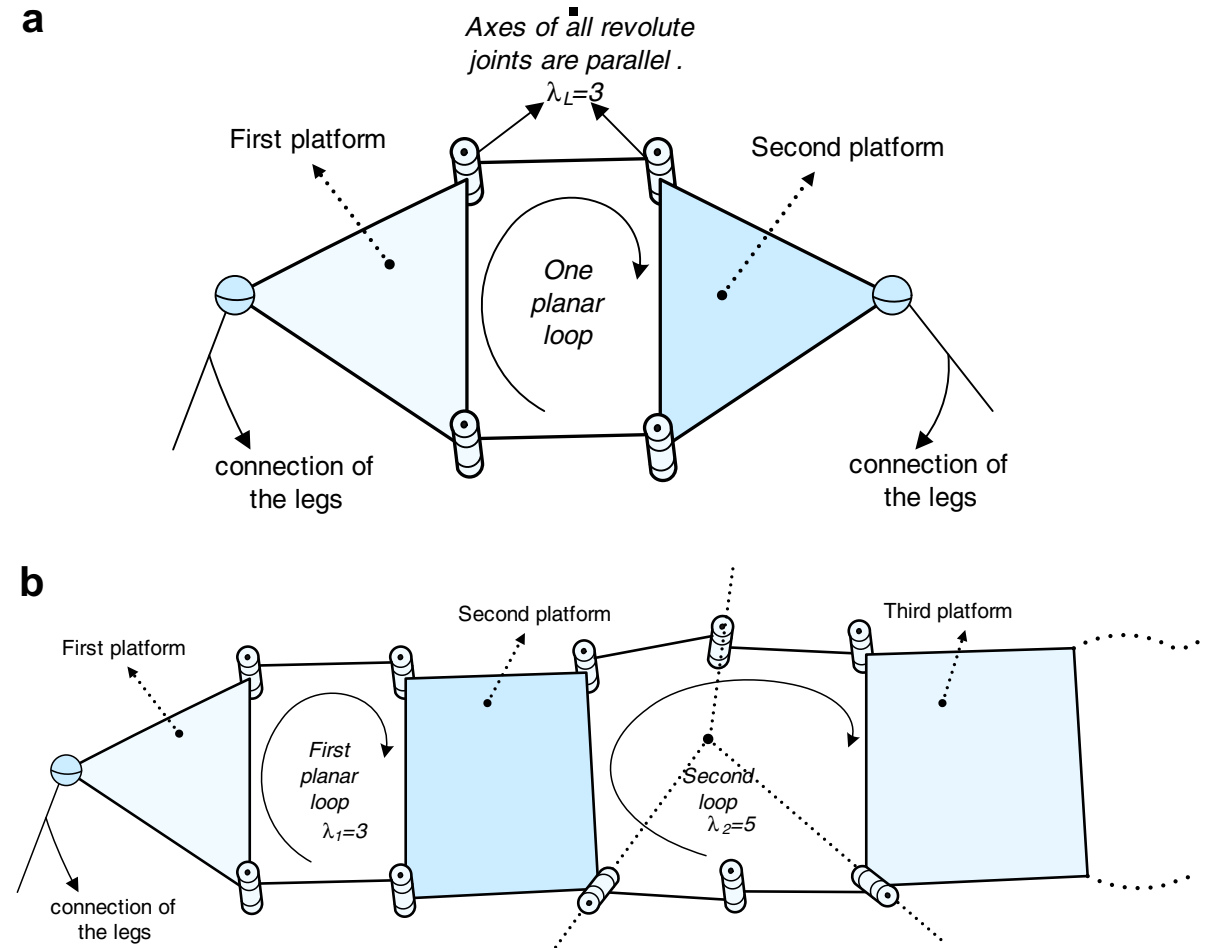

Fig. 4. (a) Two platforms connected by a planar loop. (b) Several number of loops with variable general constraints.

Example 3. Design a parallel Euclidean robot manipulator with $c_{1}=6, M=6, \lambda_{1}=6, n=2$ and $\lambda_{L}=6$. Assume that the kinematic pairs of branch-loops are configured in the same manner with kinematic chain in Fig. 5. Find the number of kinematic pairs on each leg.

By using Eq. (8), the total number of kinematic pairs on the legs can be calculated as, $\sum_{l=1}^{c_{l}} f_{l}=M-$ $\lambda-\sum_{L=1}^{n}\left(f_{L}-\lambda_{L}\right)+\sum_{l=1}^{c_{l}} \lambda_{l}=6-6-(18-12)+6 \cdot 6=30$. The numbers of kinematic pairs on each leg can be found as, $j_{l}=c_{l}^{-1} \sum_{l=1}^{6} f_{l}=5$. Kinematic structure of this manipulator is shown in Table 3. By using Eq. (9), the motion of the platforms will be $m=6$.

3.4. Structural formula of serial-parallel Euclidean platform robot manipulators that include hinges, legs and branch-loops with variable general constraints

As mentioned before, any free platform in space has six DoF. If this platform is connected to another platform by a hinge, the total DoF for two platforms will be seven. If two platforms connected by the loop which has four parallel revolute pairs, the total DoF of kinematic chain will become eight, (Fig. 6). The mobility of the legs is given in Eq. (2). Finally, the structural formula can be constructed by summing all the parameters.

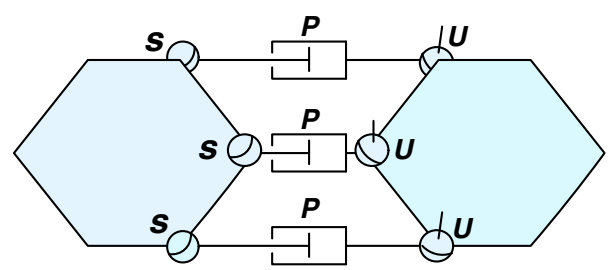

Fig. 5. Two platforms connected by two loops. 
Table 3

New parallel Euclidean platform robot manipulator that includes two branch-loops

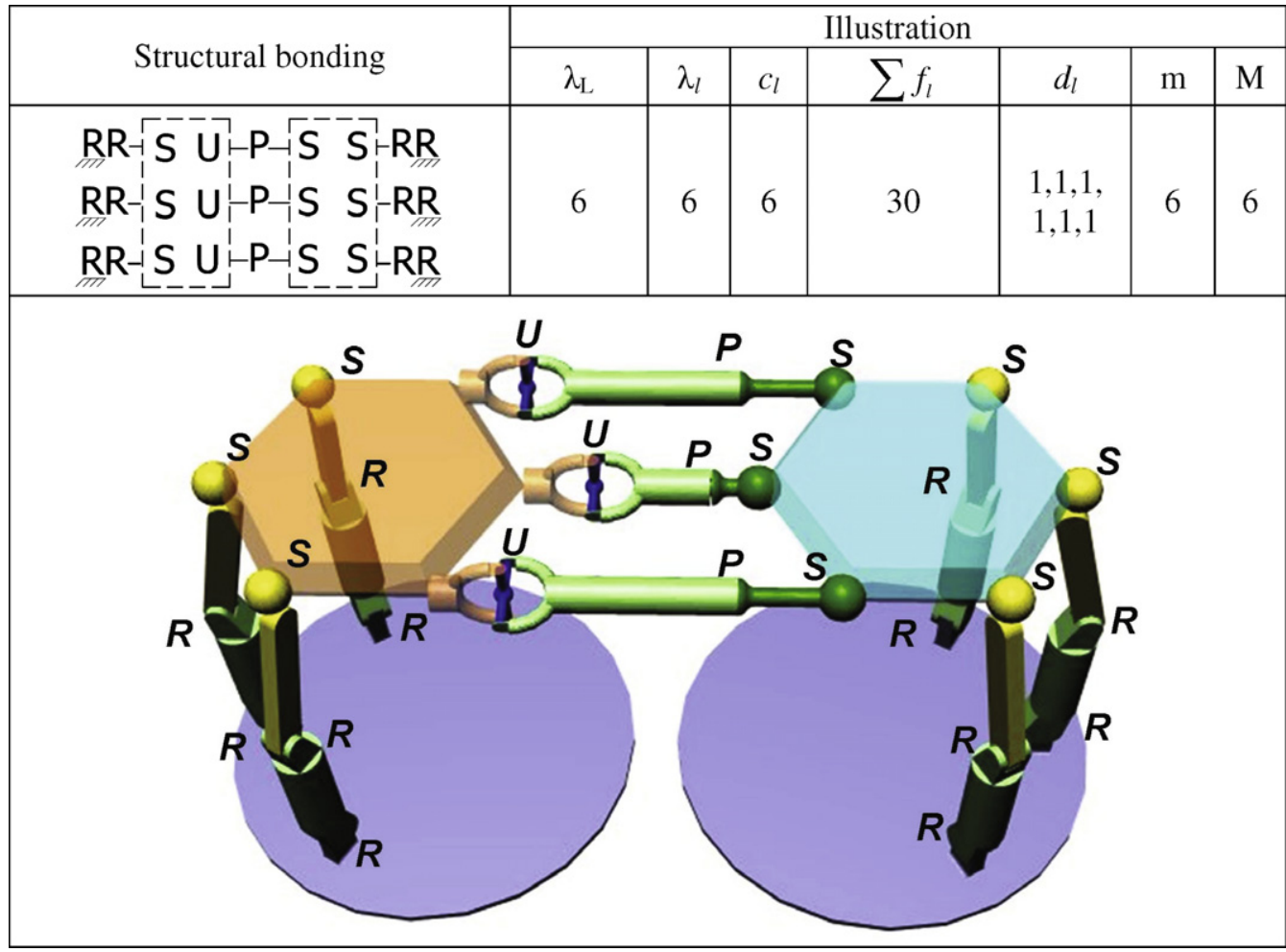

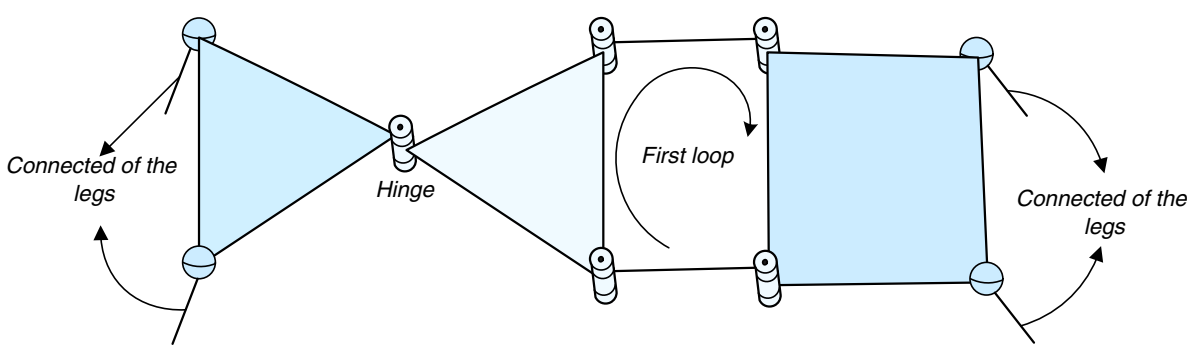

Fig. 6. Serial parallel kinematic chain created by a hinge, branch-loop and three platforms.

By combining Eqs. (3) and (8), the general structural formula of serial-parallel Euclidean robot manipulators with variable general constraints that include, several hinges, legs and branch-loops can be defined as,

$$
M=\lambda+j_{h}+\sum_{L=1}^{n}\left(f_{L}-\lambda_{L}\right)+\sum_{l=1}^{c_{l}}\left(f_{l}-\lambda_{l}\right)
$$

The general formula of motion of platforms that are created by branch-loops and hinges can be given in the following form.

$$
m=\lambda+c_{l}+\sum_{l=1}^{c_{l}}\left(d_{l}-D\right)+j_{h}+\sum_{L=1}^{n}\left(f_{L}-\lambda_{L}\right)
$$




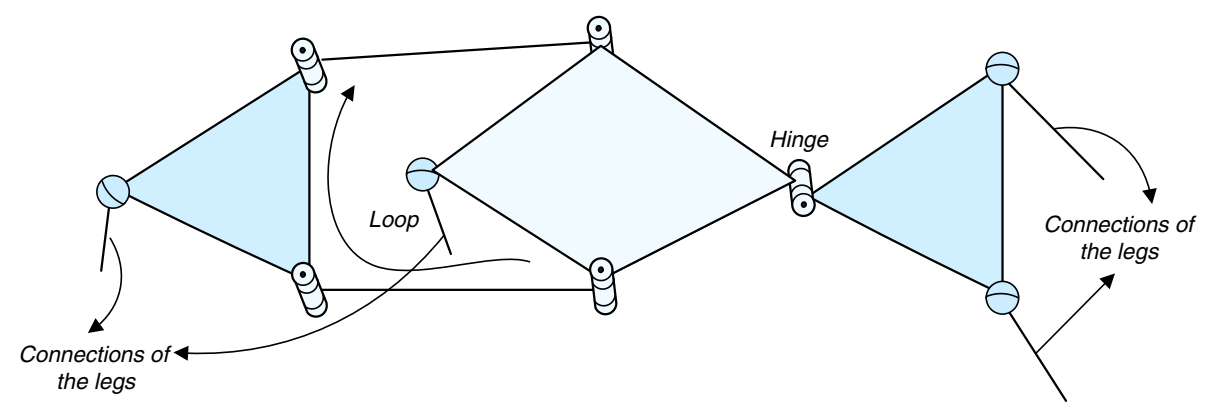

Fig. 7. Configuration of kinematic pairs of the branch-loop and hinge.

Table 4

New serial-parallel Euclidean platform robot manipulator with one loop and hinge

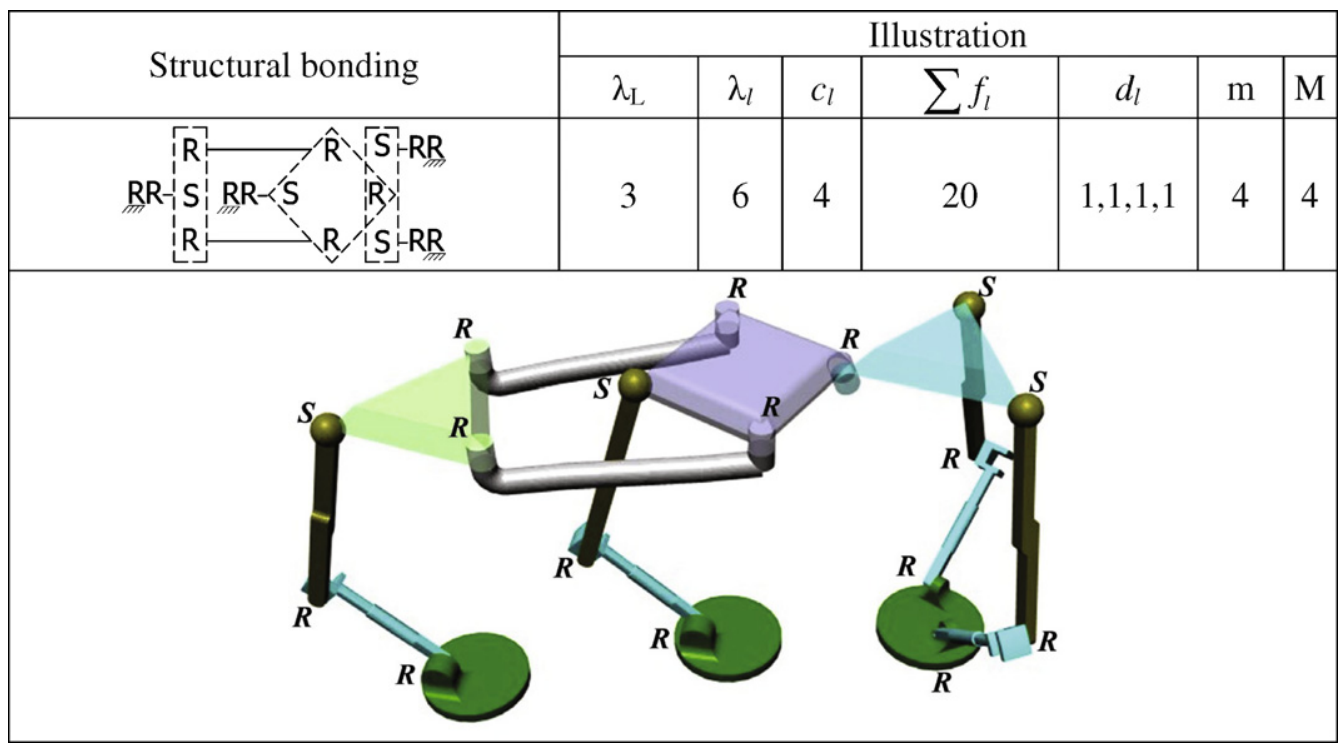

Example 4. Design a serial Euclidean robot manipulator with $c_{1}=4, M=4, \lambda_{1}=6, n=1, \lambda_{L}=3$ and $j_{h}=1$. Assume that kinematic pairs of the loop and hinge are configured as kinematic chain in Fig. 7. Find the number of kinematic pairs on each leg.

By using Eq. (10), the total degrees of freedom of the legs can be found as, $\sum_{l=1}^{c_{l}} f_{l}=M-\lambda-j_{h}-$ $\sum_{L=1}^{n}\left(f_{L}-\lambda_{L}\right)+\lambda_{l} \cdot c_{l}=4-6-1-1+6 \cdot 4=20$, and the number of kinematic pairs on each leg will be $j_{l}=c_{l}^{-1} \sum_{l=1}^{4} f_{l}=5$. Using exchangeability of kinematic pairs, the new Euclidean serial-parallel manipulator can be designed as shown in Table 4. The motion of platforms can be calculated by using Eq. (11) as, $m=\lambda+c_{l}+\sum_{l=1}^{c_{l}}\left(d_{l}-D\right)+j_{h}+\sum_{L=1}^{n}\left(f_{L}-\lambda_{L}\right)=6+4+(1-3)+(1-3)+(1-3)+(1-3)+1+(4-3)=4$.

\section{Classification of simple platform structural groups with variable general constraints}

To create new robot manipulators, simple platform structural groups with variable general constraints, platforms, hinges, legs and branch loops from different subspaces and space should be considered. By definition, simple structural groups can not be split into other structural groups and their mobility should be equal to zero $(M=0)$ as no input parameters exist. As a result, the general mobility Eq. (10) can be described as, 
Table 5

One DoF branch loops with variable general constraints

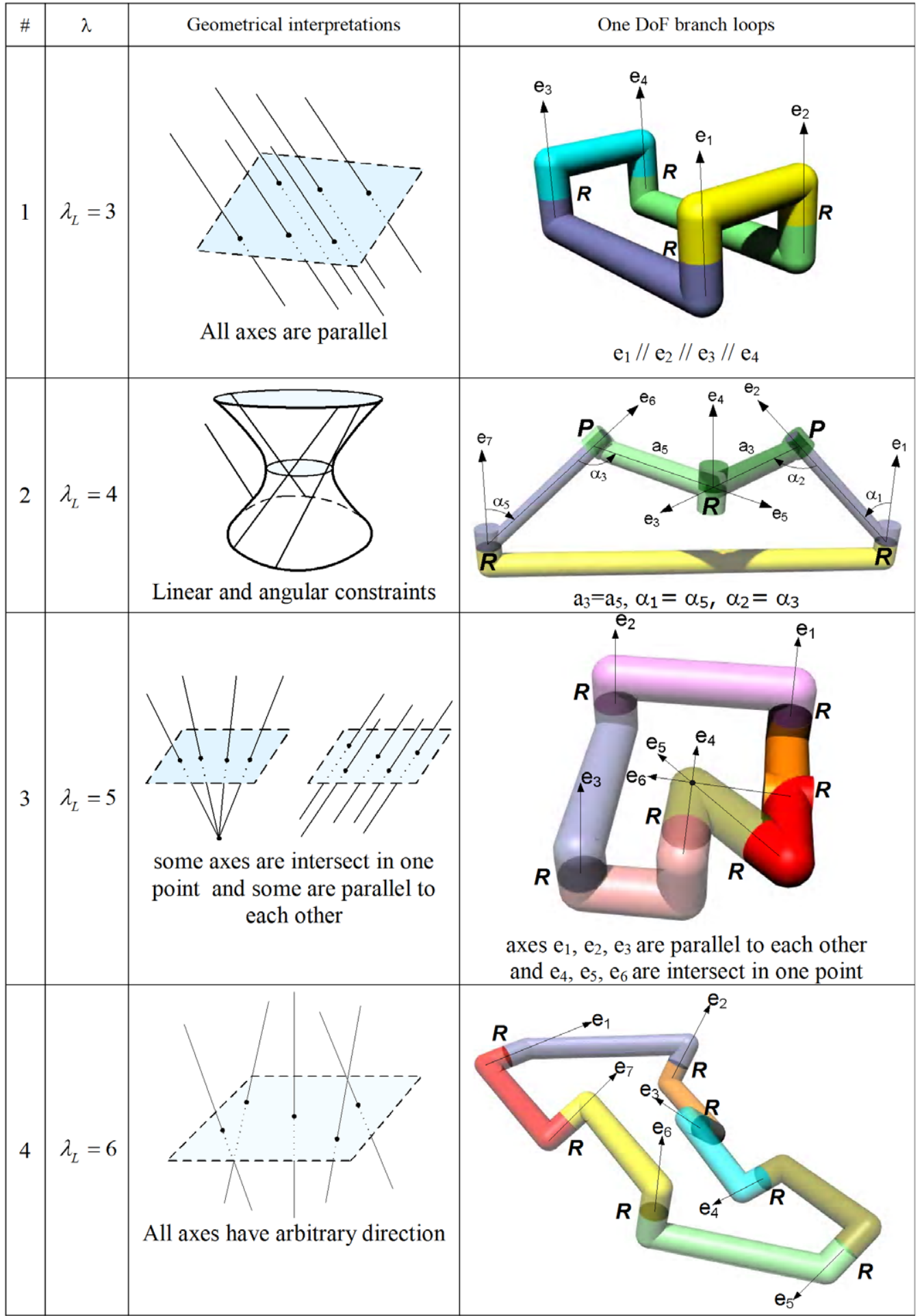


Table 6

Simple platform structural groups with variable general constraints

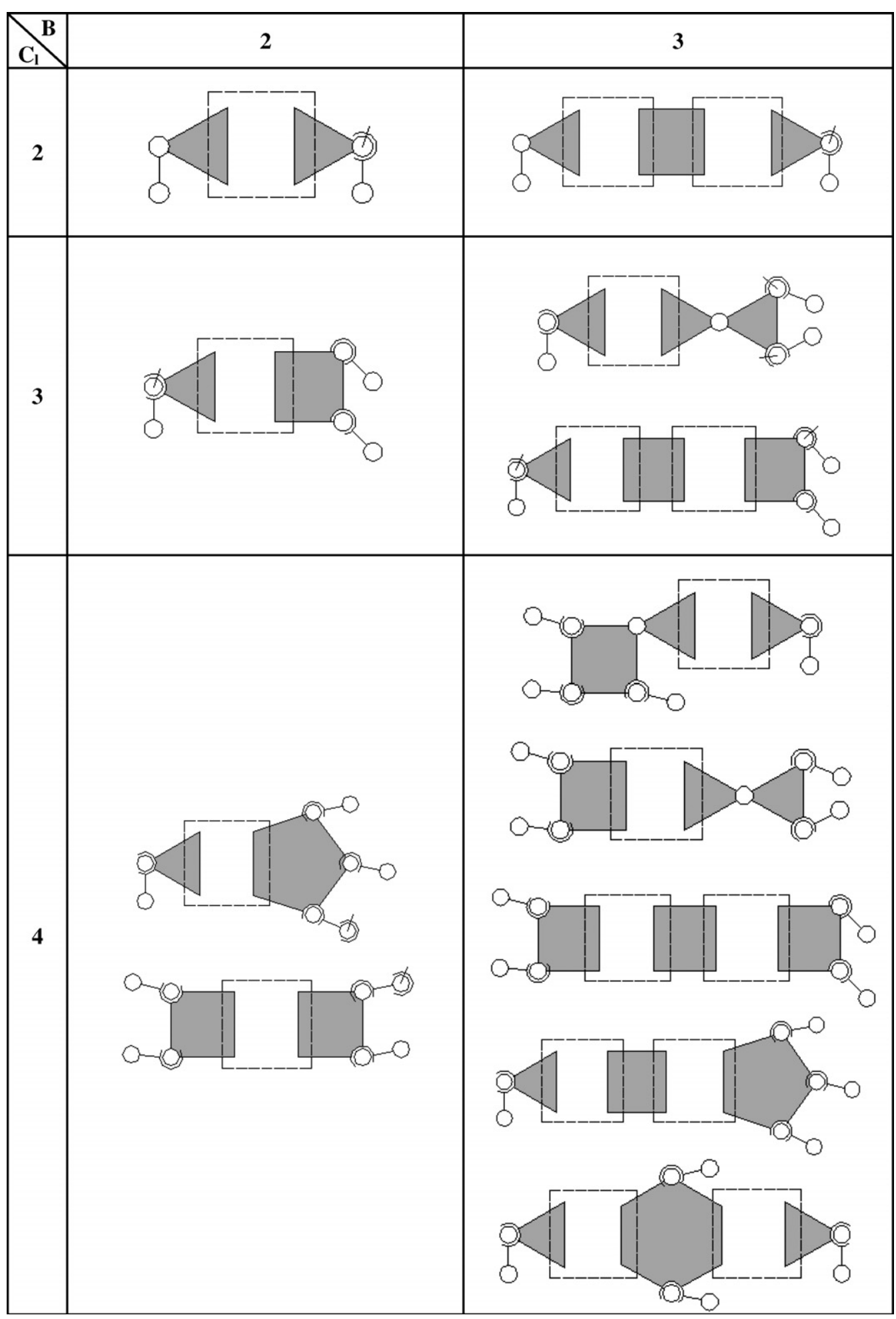




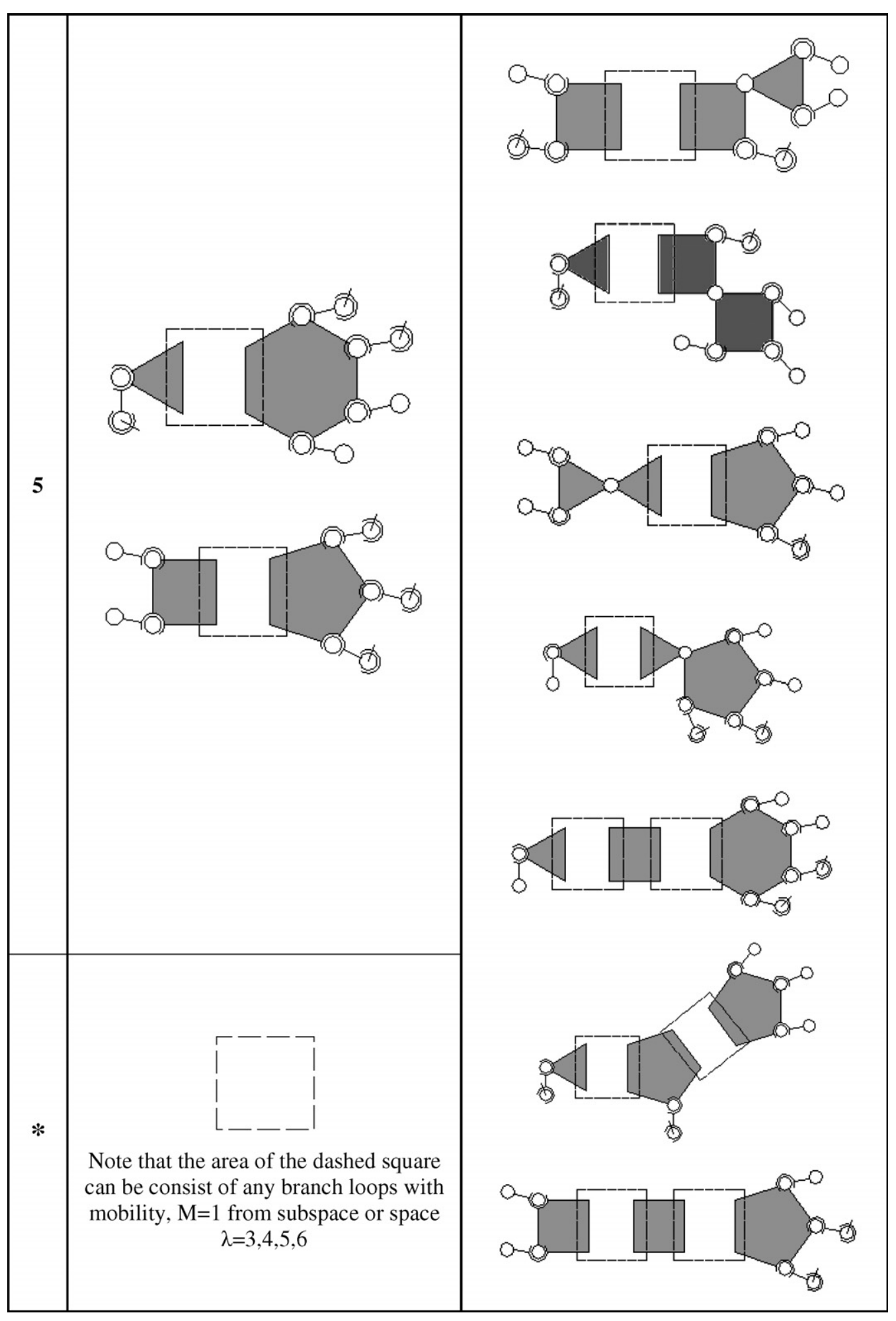




$$
\lambda+j_{h}+\sum_{L=1}^{n}\left(f_{L}-\lambda_{L}\right)+\sum_{l=1}^{c_{l}}\left(f_{l}-\lambda_{l}\right)=0
$$

Using Eq. (12), the total number of joints on the legs of simple platform structural groups with variable general constraints for $\lambda=6$, and $\lambda_{1}=6$ can be introduced as,

$$
\sum_{l=1}^{c_{l}} f_{l}=6\left(c_{l}-1\right)-\sum_{L=1}^{n}\left(f_{L}-\lambda_{L}\right)-j_{h}
$$

If all the branch loops with variable general constraints are considered in minimum mobility $(M=1)$, the value of the term $\left(f_{L}-\lambda_{L}\right)$ will be unity. So that the Eq. (13) can be rewritten as an objective function of structural synthesis as,

$$
\sum_{l=1}^{c_{l}} f_{l}=6\left(c_{l}-1\right)-n-j_{h}
$$

The additional conditions in the structural synthesis of platform structural groups with variable general constraints can be expressed as following equality and inequality constraints:
(a) $B \geqslant 2$
(b) $3 \leqslant j_{B} \leqslant 6$
(c) $j_{h}+n=B-1$
(d) $c_{l}=j_{B}-2 j_{h}-4 n$
(e) $j_{l}=c_{l}^{-1} \sum f_{l}$

where $B$ represents the number of moving platforms, $j_{B}$ introduces the number of joints on the moving platforms and $n$ is the number of branch-loops.

Table 7

New advanced serial-parallel Euclidean platform robot manipulator

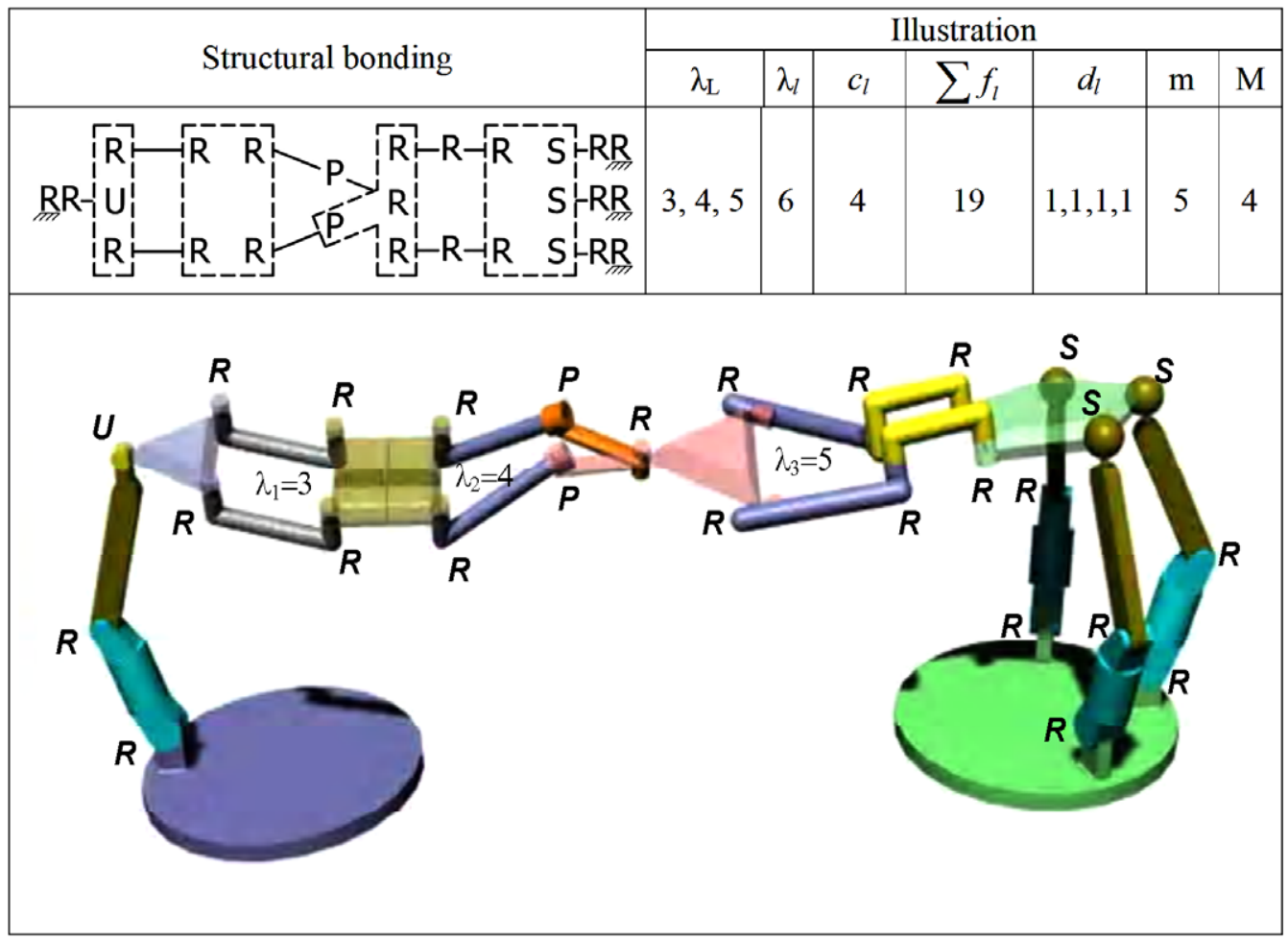


Table 8

Classification of simple platform structural groups with variable general constraints

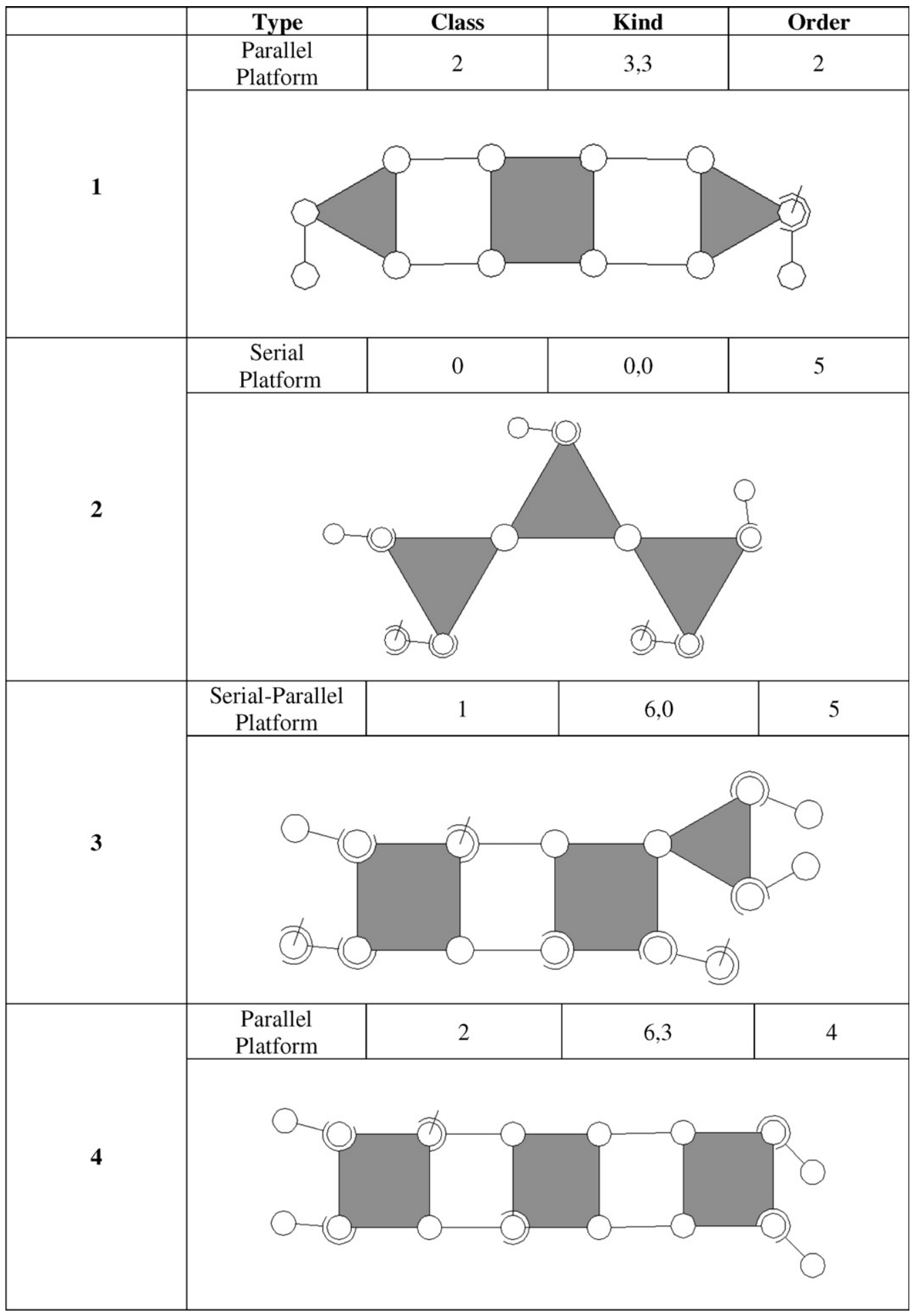


Using the objective function in Eq. (14) and equality-inequality constraint conditions (Eq. (15)), a computer software of structural synthesis of simple platform structural groups with variable general constraints has been created. The algorithm of structural synthesis can be summarized step by step as follows:

- Select values for $B$ and $j_{B}$ (Eqs. (15a) and (15b)).

- Select the values for $j_{h}$ and $n$ with respect to Eq. (15c).

- Calculate the number of legs $c_{1}$ Eq. ((15d)).

- Calculate the total number of 1 DoF joints $\sum f_{l}$ (Eq. (14)).

- Place the joints on legs (Eq. (15e)), branch loops and hinges. Note that branch loops can be selected from any subspace or space $3 \leqslant \lambda \leqslant 6$ provided that mobility of the loop is unity $(M=1)$ (Table 5 ).

- Using the exchangeability principle, 1 DoF joints can be replaced by higher or other kinematic pairs.

- Mobility of the manipulator will be equal to the number of actuators added to simple platform structural group.

Some results for simple platform structural groups are presented in Table 6.

Also, another new advanced serial-parallel Euclidian platform robot manipulator with variable general constraints can be introduced in Table 7. In the design of this manipulator, three branches loops are selected from Table 5 .

The following definitions for simple structural groups with variable general constraints can be extracted from the equality-inequality constraints as,

- The number of platforms are equal to the sum of the number of hinges and branch-loops plus one, $B=j_{h}+n+1$.

- Each branch loop will at least take four joints from the connecting platforms.

- Each hinge will take two joints from the connecting platforms.

- Number of the legs is equal to the difference between the total joints on the platforms and the total joints taken by hinges and loops for connections.

Being able to create the simple platform structural groups with variable general constraints, following definitions can be given for structural classification:

- Type of the simple platform structural groups is expressed by three variations; Serial Platforms, where the platforms are connected to each other only by hinge joints, Parallel Platforms, where the platforms are connected to each other only by branch loops, and Serial-Parallel Platforms, where the platforms are connected to each other by both hinges and branch loops.

- Class of the simple platform structural groups is expressed by the number of branch loops.

- Kind of the simple platform structural groups is expressed by the variable general constraints of the branch loops.

- Order of the simple platform structural groups is expressed by the number of legs.

A sample classification of the selected simple platform structural groups with variable general constraints is given in Table 8.

Table 9

New mobility formulations

\begin{tabular}{llllll}
\hline $\begin{array}{l}\text { Mobility of } \\
\operatorname{legs}\left(M_{\mathrm{l}}\right)\end{array}$ & $\begin{array}{l}\text { Mobility of single } \\
\text { platform }\left(M_{\mathrm{P}}\right)\end{array}$ & $\begin{array}{l}\text { Mobility of } \\
\text { hinges }\left(M_{\mathrm{H}}\right)\end{array}$ & $\begin{array}{l}\text { Mobility of branch- } \\
\operatorname{loops}\left(M_{\mathrm{BL}}\right)\end{array}$ & $\begin{array}{l}\text { Mobility equation } \\
M=M_{1}+M_{\mathrm{p}}+M_{\mathrm{H}}+M_{\mathrm{BL}}\end{array}$ \\
\hline 1 & $\sum_{l=1}^{c_{l}}\left(f_{l}-\lambda_{l}\right)$ & $\lambda$ & - & - & $M=\lambda+\sum_{l=1}^{c_{l}}\left(f_{l}-\lambda_{l}\right)$ \\
2 & $\sum_{l=1}^{c_{l}}\left(f_{l}-\lambda_{l}\right)$ & $\lambda$ & $j_{h}$ & - & $M=\lambda+j_{h}+\sum_{l=1}^{c_{l}}\left(f_{l}-\lambda_{l}\right)$ \\
3 & $\sum_{l=1}^{c_{l}}\left(f_{l}-\lambda_{l}\right)$ & $\lambda$ & - & $\sum_{L=1}^{n}\left(f_{L}-\lambda_{L}\right)$ & $M=\lambda+\sum_{L=1}^{n}\left(f_{L}-\lambda_{L}\right)+\sum_{l=1}^{c_{l}}\left(f_{l}-\lambda_{l}\right)$ \\
4 & $\sum_{l=1}^{c_{l}}\left(f_{l}-\lambda_{l}\right)$ & $\lambda$ & $j_{h}$ & $\sum_{L=1}^{n}\left(f_{L}-\lambda_{L}\right)$ & $M=\lambda+j_{h}+\sum_{L=1}^{n}\left(f_{L}-\lambda_{L}\right)+\sum_{l=1}^{c_{l}}\left(f_{l}-\lambda_{l}\right)$ \\
\hline
\end{tabular}

(1) Parallel EPRM; (2) Serial EPRM; (3) Parallel EPRM with branch-loops; (4) Serial-parallel EPRM. 


\section{Conclusion}

Kinematic analysis of robot manipulators can be a difficult and complex task depending on the DoF concept. However, by selecting the legs of the robot manipulators as moving dyads on Euclidean planes, these analyses will be simplified, due to the fact that, the direct and inverse tasks will become easier to solve. In the light of this, in this study, new structural formulations are presented to design various new parallel Euclidean robot manipulators with variable general constraints. With respect to the new formulations (Table 9), new serial, parallel, and serial-parallel Euclidean platform manipulators are created and explained along with examples. Also their illustrations are presented in the tables including structural parameters, structural bondings and kinematic structures. Moreover, definitions and classifications for simple structural groups with variable general constraints are introduced. Finally, by using systematic process of structural synthesis, an algorithm is developed to create new structures of serial, parallel, and serial-parallel Euclidean platform robot manipulators.

\section{References}

[1] T.S. Mruthyunjaya, Kinematic structure of mechanisms revisited, Mech. Mach. Theory 38 (2003) 279-320.

[2] G. Gogu, Mobility of mechanisms: a critical review, Mech. Mach. Theory 40 (2005) 1068-1097.

[3] R. Alizade, Ç. Bayram, E. Gezgin, Structural synthesis of serial platform manipulators, Mech. Mach. Theory 42 (2007) 580-599.

[4] J. Denavit, R.S. Hartenberg, A kinematic notation for lower pair mechanisms based on matrices, J. Appl. Mech. 22 (1955) $215-221$.

[5] N.P. Sheth, J.J. Uicker, A generalized symbolic notation for mechanism, J. Eng. Ind. - Trans. ASME 93 (1971) $102-112$.

[6] W. Khalil, J. Kleifinger, Nouvelles notations pour decrire la structure géometrique des robots, Note interne, No 05/85, LAN Nantes, 1985.

[7] R. Alizade, Ç. Bayram, Structural synthesis of parallel manipulators, Mech. Mach. Theory 39 (2004) 857-870.

[8] B. Roth, Performance Evaluation of Manipulators from a Kinematic Viewpoint, vol. 459, NBS Special Publications, 1975 , pp. 39-61.

[9] F.R.E. Crossley, A contribution to Grubler's theory in the number synthesis of plane mechanisms, J. Eng. Ind - Trans. ASME 86/01 (1964) $1-8$.

[10] L.S. Woo, Type synthesis of plane linkages, J. Eng. Ind. - Trans. ASME B-89 (1967) 159-172.

[11] T.S. Mruthyunjaya, Structural synthesis by transformation of binary chains, Mech. Mach. Theory 14 (1979) 221-238.

[12] T.S. Mruthyunjaya, A computerized methodology for structural synthesis of kinematic chains: Part 1 - formulation, Mech. Mach. Theory 19 (6) (1984) 487-495.

[13] T.S. Mruthyunjaya, A computerized methodology for structural synthesis of kinematic chains: Part 2 - application to several fully or partially known cases, Mech. Mach. Theory 19 (6) (1984) 497-505.

[14] N. Manolescu, A unified method for the formulation of all planar joined kinematic chains and Baranov trusses, Environ. Plann. B6 (1979) 447-454.

[15] N. Manolescu, Sur la structure des mécanismes en robotique, in: Conférence á í Ecole centrale darts et manufactures, Paris, 1987.

[16] N. Manolescu, I. Tudosie, I. Balanescu, D. Birciu, T. Lonescu, Structural and kinematic synthesis of planar kinematic chain (PKC) and mechanisms (PM) with variable structure during the work, in: Proceedings of 7 th World Congress, The Theory Machines \& Mechanics, Sevilia, Spain, 1987, pp. 45-48.

[17] J.P. Merlet, Les robots paralléles, Hérmes, Paris, 1990, p. 345.

[18] W.M. Hwang, Y.W. Hwang, Computer-aided structural synthesis of planar kinematic chains with simple joints, Mech. Mach. Theory 27 (2) (1992) 189-199.

[19] P. Mitrouchev, Symbolic structural synthesis and a description method for planar kinematic chains in robotics, Eur. J. Mech. A/ Solids 20 (2001) 777-794.

[20] A.C. Rao, P.B. Deshmukh, Computer aided structural synthesis of planar kinematic chains obviating the test for isomorphism, Mech. Mach. Theory 36 (2001) 489-506.

[21] J.-S. Zhao, K. Zhou, Z.-J. Feng, A theory of degrees of freedom for mechanism, Mech. Mach. Theory 39 (2004) $621-643$.

[22] Z. Huang, Q.C. Li, General methodology for type synthesis of symmetrical lower-mobility parallel manipulators and several novel manipulators, Int. J. Robot. Res. 21 (2002) 131-145.

[23] T. Huang, M. Li, X.M. Zhao, J.P. Mei, D.G. Chetwynd, S.J. Hu, Conceptual design and dimensional synthesis for a 3-DOF module of the trivariant - a novel 5-DOF reconfigurable hybrid robot, IEEE Trans. Robot. 21 (3) (2005) 449-456.

[24] Xin-Jun Liu, Jinsong Wang, Gunter Pritschow, A new family of spatial 3-DoF fully-parallel manipulators with high rotational capability, Mech. Mach. Theory 40 (2005) 475-494.

[25] Xin-Jun Liu, Jinsong Wang, Some new parallel mechanisms containing the planar four-bar parallelogram, Int. J. Robot. Res. 22 (2003) 717-732.

[26] Yuefa Fang, Lung-Wen Tsai, Structure synthesis of a class of 4-DoF and 5-DoF parallel manipulators with identical limb structures, Int. J. Robot. Res. 21 (2002) 799-810.

[27] J.M. Herve, The Lie group of rigid body displacements a fundamental tool for mechanism design, Mech. Mach. Theory 34 (1999) 719-730.

[28] A. Gray, Modern Differential Geometry of Curves and Surfaces, CRC Press, 1993, p. 664. 